\title{
Design of a Integrated readout Circuit for pH Sensor with Chopper Instrumentation Amplifier
}

\author{
Yang Wang ${ }^{1, a}$, Jiang $\mathrm{Hu}^{1, b}$ and Zongqi Guan ${ }^{1, \mathrm{c}}$ \\ ${ }^{1}$ Department of Electronics and Information, Zhejiang Wanli University, Ningbo 315100, P. R. China \\ awangyang1028@126.com, bhujiang0220@163.com, 'cguanzongqi@yeah.net
}

Keywords: pH sensor; readout circuit; CMOS; chopper stabilization; instrumentation amplifier Abstract. A readout circuit for capacitive $\mathrm{pH}$ sensor is designed and simulated by Spectre of Cadence software with TSMC $0.18 \mu \mathrm{m}$ CMOS process technology. It consists of a capacitance-voltage measuring circuit, a modulation and demodulation circuit, a instrumentation amplifier and a low-pass filter. The simulation results show that the sensitivity is $10.8 \mathrm{mV} / \mathrm{fF}$ and the circuit reduces the low frequency noise and DC offset using the technique of chopper instrumentation amplifier.

\section{Introduction}

Micro-electro-mechanical system (MEMS) technology has become popular for the miniaturization of sensors. With the rapid development of complementary metal oxide semiconductor (CMOS) technology, the integration of sensing circuit and MEMS transducer on the chip has some advantages including small size, easy mass-production and low cost. Recently, various CMOS-MEMS sensors have been used in agriculture, environmental, food industry, home appliances, etc[1,2].

$\mathrm{pH}$ value is one of the most common measurement parameters because chemical and biological processes are dependent on $\mathrm{pH}$. For several decades, the $\mathrm{pH}$ ion-sensitive field effect transistors (ISFET) has been receiving more attention since ISFET were first demonstrated by Bergveld in 1970s[3]. pH-ISFET use potentiometric method to detect the change in $\mathrm{pH}$ through the corresponding shift in the threshold voltage[4,5]. Capacitive $\mathrm{pH}$ sensors have advantages over $\mathrm{pH}$-ISFET of smaller size, less noise and higher ability with integrated CMOS-MEMS process. The principle of them is to measure capacitance variances with $\mathrm{pH}$ value changes of waters, rivers and soils[6].

However, there are challenges of detecting ultra-low sensing capacitance for integrated capacitive $\mathrm{pH}$ sensor. Hence, different means including correlated double sampling(CDS) and chopper stabilization are implemented to reduce low frequency noise and DC offset in amplifiers in the readout circuit of integrated capacitive sensors[7,8].

In this paper, a readout circuit for capacitive $\mathrm{pH}$ sensor is designed and implemented based on the technique of chopper stabilization and instrumentation amplifier. The circuit is simulated and confirmed by Cadence software and CMOS process technology.

\section{Proposed integrated readout circuit architecture}

Chopper stabilization is a commonly used method to read a small capacitance value for capacitive sensors. It works based on modulation and demodulation principles, as shown in Fig. 1. The low frequency input signal is modulated, amplified and then demodulation back to DC which the flicker noise and offset is modulated to high frequency[9]. The high frequency component is suppressed by a low-pass filter.
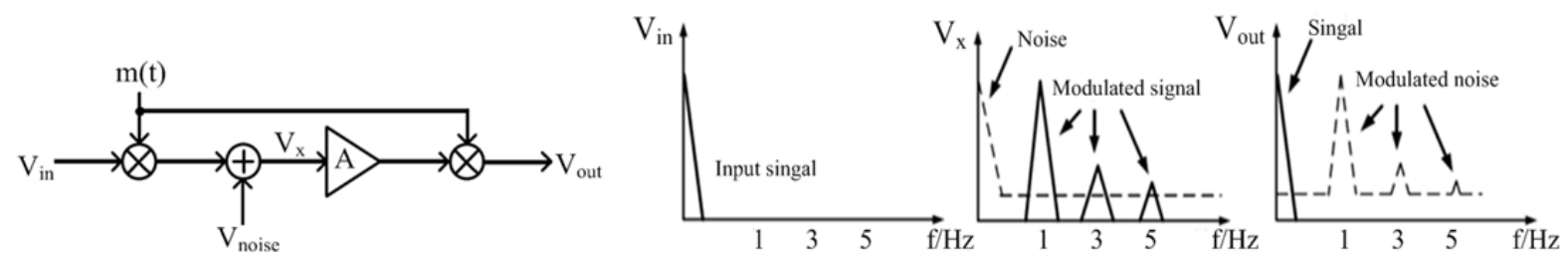

Fig. 1 Chopper amplifier principle 
Instrumentation amplifier has a large input impedance, high common-mode rejection ration, and precise gain, which is applied as capacitive sensor front-end circuits[10]. Fig. 2 shows a typical instrumentation amplifier architecture.

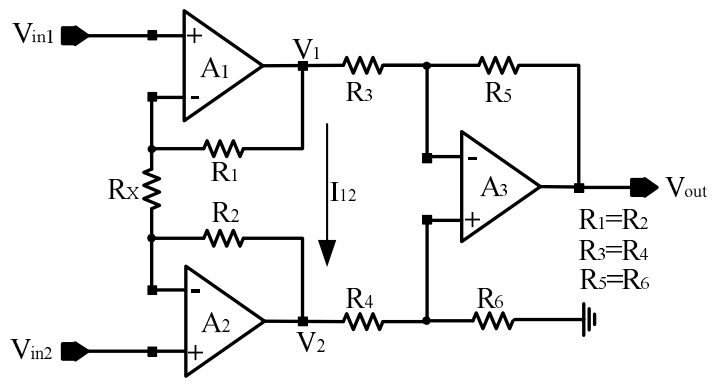

Fig. 2 Typical architecture of instrumentation amplifier

$$
\begin{aligned}
& V_{1}-V_{2}=I_{12}\left(R_{X}+2 R_{1}\right)=\frac{V_{i n 1}-V_{i n 2}}{R_{X}}\left(R_{X}+2 R_{1}\right) \\
& V_{\text {out }}=-\frac{R_{5}}{R_{3}}\left(V_{1}-V_{2}\right)=-\frac{R_{5}}{R_{3}}\left(1+\frac{2 R_{1}}{R_{X}}\right)\left(V_{i n 1}-V_{\text {in } 2}\right)
\end{aligned}
$$

Therefore, the aim of this study is to design and implement an chopper-stabilized instrumentation amplifier circuit for capacitive $\mathrm{pH}$ sensor. The readout circuit is shown as Fig. 3, which provides a good low-frequency noise and low offset characteristic. The circuit consists of a capacitance-voltage measuring circuit, a modulation and demodulation circuit, a instrumentation amplifier and a low-pass filter.

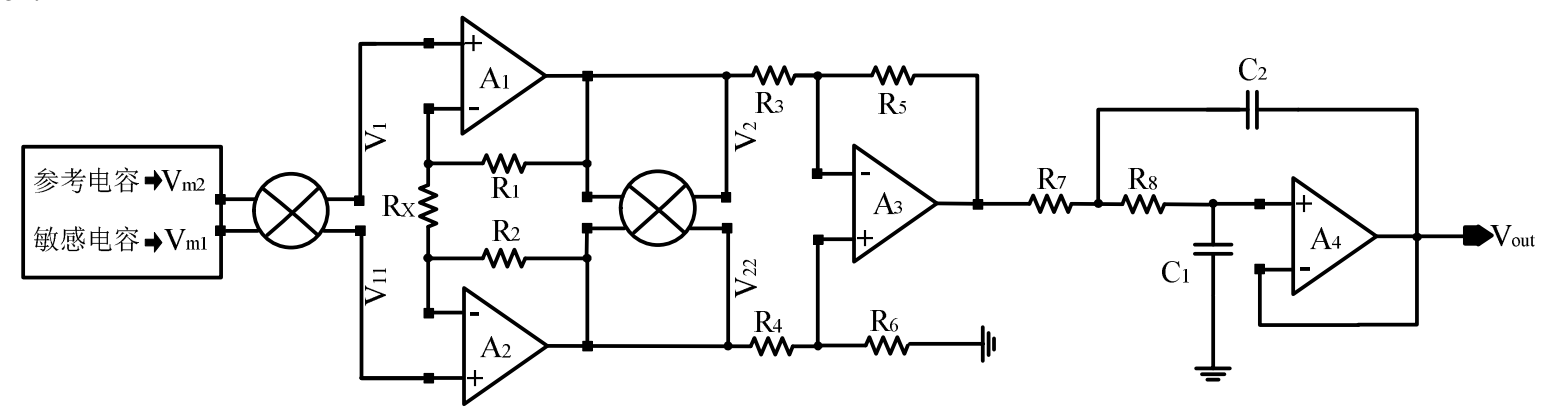

Fig. 3 Block diagram of the readout circuit

\section{Operation and design of readout circuit}

Integrated capacitive pH sensor with comb electrode. The sensitive capacitance of the capacitive $\mathrm{pH}$ sensor with comb electrodes alters with $\mathrm{pH}$ value change. $L$ is the length of comb electrodes, $s$ is the width of each electrode, and $g$ is the distance between adjacent electrodes. The thickness of the sensing material layer and the electrode is $h$ and $t$, as shown in Fig. 4. The top sensing material layer is the silicon nitride to provide the sensing membrane for $\mathrm{pH}$ buffer solutions[6].
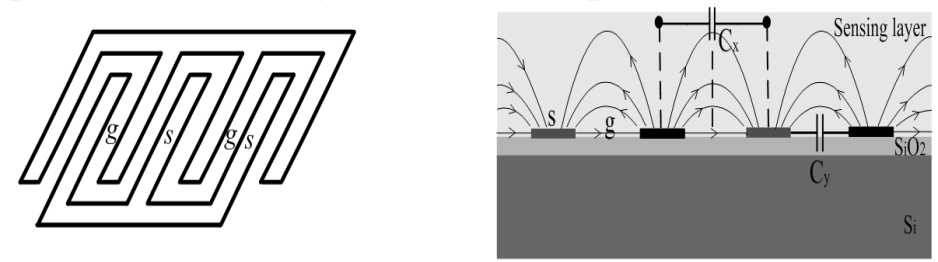

Fig. 4 Structure of a capacitive $\mathrm{pH}$ sensor and equivalent capacitors

$C_{x}$ is the capacitance of one electrode and the group in the sensitive layer. Based on conformal mapping techniques[11], the variation of the $C_{x}$ is given by

$$
\Delta C_{x}=\frac{1}{2} \cdot \varepsilon_{0} \cdot \Delta \varepsilon_{p H} \cdot \frac{L \cdot K\left(k^{\prime}\right)}{K(k)}
$$

Where $K(\cdot)$ is the complete elliptic integral of first kind, $k$ is the modulus, $k^{\prime}$ is the complementary modulus of $k$, and $\Delta \varepsilon_{p H}$ is the change in the relative permittivity of $\mathrm{pH}$ sensing membrane. 


$$
\begin{aligned}
& k=\sin \left(\pi \cdot \frac{g}{2(s+g)}\right) \\
& k^{\prime}=\cos \left(\pi \cdot \frac{g}{2(s+g)}\right)
\end{aligned}
$$

The change of the capacitance $\Delta C_{y}$ can be computed as parallel plane capacitors

$$
\Delta C_{y}=\varepsilon_{0} \cdot \Delta \varepsilon_{p H} \cdot \frac{L \cdot t}{g}
$$

Therefore, the change of $\mathrm{pH}$ sensor capacitance $\Delta C_{S}$ is described by

$$
\Delta C_{s}=\Delta C_{x}+\Delta C_{y}=(n-1) \cdot \varepsilon_{0} \cdot \Delta \varepsilon_{p H} \cdot L \cdot\left[\frac{K\left(k^{\prime}\right)}{2 \cdot K(k)}+\frac{t}{g}\right]
$$

Capacitance-voltage measuring circuit. The capacitance-voltage measuring circuit is designed based on the principle of current mirror as shown in Fig. 5. Using the charge-discharge of sensing capacitor, the voltages change with varies the sensitive capacitance is measured[12].

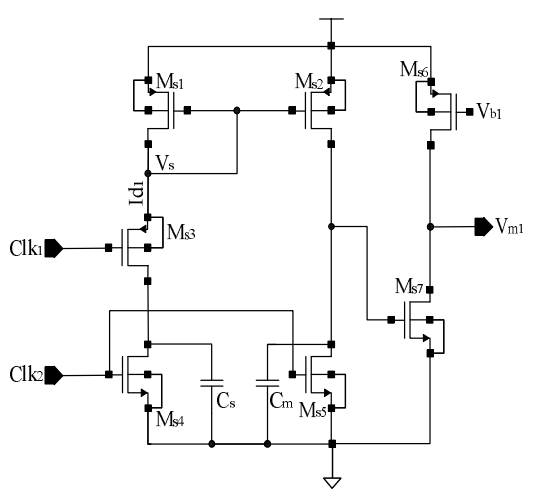

Fig. 5 capacitance-voltage measuring circuit

If the effect of channel length modulation is ignored and $M_{s 1}$ is saturated, the voltage of sensing capacitor can be expressed as

$$
\begin{aligned}
& C_{\mathrm{s}} \frac{d V_{s}}{d t}=I_{d 1}=\frac{1}{2} u_{p} C_{o x}\left(\frac{W}{L}\right)_{s 1}\left(V_{d d}-V_{s}-V_{p t h}\right)^{2} \\
& V_{s}=V_{d d}-V_{p h t}-\frac{1}{\frac{1}{2} u_{p} C_{o x}\left(\frac{W}{L}\right)_{s 1} \frac{1}{C_{s}} t+\frac{1}{V_{d d}-V_{p h h}}} \\
& M_{s 1} \text { and } M_{s 2} \text { is the relation of the current mirror. If } L_{s 1}=L_{s 2} \\
& V_{m 1}=\left(\frac{W}{L}\right)_{s 2}\left(\frac{L}{W}\right)_{s 1} \frac{C_{s}}{C_{m}} V_{s}=\frac{W_{s 2}}{W_{s 1}} \frac{C_{s}}{C_{m}}\left[V_{d d}-V_{p h t}-\frac{1}{\left.\frac{1}{2} u_{p} C_{o x}\left(\frac{W}{L}\right)_{s 1} \frac{1}{C_{s}} t+\frac{1}{V_{d d}-V_{p h h}}\right] \approx \frac{W_{s 2}}{W_{s 1}}\left(V_{d d}-V_{p h h}\right) \frac{C_{s}}{C_{m}}}\right.
\end{aligned}
$$

In Fig. 5, $V_{m 2}$ can be obtained when $C_{s}$ is replaced by $C_{r e f}$. Therefore,

$$
V_{m 1}-V_{m 2}=\frac{W_{s 2}}{W_{s 1}}\left(V_{d d}-V_{p h h}\right) \frac{C_{s}-C_{r f}}{C_{m}}=\frac{W_{s 2}}{W_{s 1}}\left(V_{d d}-V_{p h h}\right) \frac{\Delta C_{s}}{C_{m}}
$$

Chopper circuit. The differential chopper is composed of transmission gates as shown in Fig. 6. The transmission gates are controlled by differential clock.

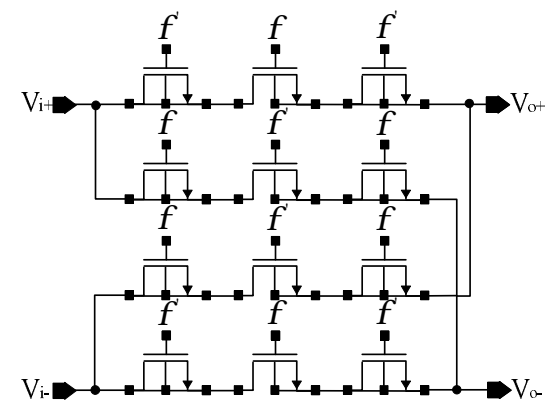

Fig. 6 chopper circuit 
The carrier modulation function of transmission gates is

$$
\begin{aligned}
& m(t)=\left\{\begin{array}{l}
+1,0<t<\frac{T}{2} \\
-1, \frac{T}{2}<t<T
\end{array}\right. \\
& f[m(t)]=a_{0}+\sum_{n=1}^{n=\infty}\left\{a_{n} \cos \frac{2 n \pi}{T}+b_{n} \sin \frac{2 n \pi}{T}\right\} \\
& a_{0}=\frac{1}{T} \int_{0}^{T} m(t) d t=0 \\
& a_{n}=\frac{2}{T} \int_{0}^{T} m(t) \cos \frac{2 n \pi}{T} t d t=0 \\
& b_{n}=\frac{2}{T} \int_{0}^{T} m(t) \cdot \sin \frac{2 n \pi}{T} t d t=\frac{4}{\pi} \cdot \frac{1}{n} \\
& f[m(t)]=\frac{4}{\pi}\left\{\sin \omega t+\frac{1}{3} \sin 3 \omega t+\frac{1}{5} \sin 5 \omega t+\ldots\right\}
\end{aligned}
$$

Therefore, the input signal is modulated to a higher chopping frequency, and the noise and offset are removed using a low-pass filter when the demodulator demodulates back the signal to the baseband.

Telescopic cascode operational amplifier circuit. The two-stage operational amplifier with miller frequency compensation is designed. The first stage is a telescopic cascode amplifier and a second stage is a output amplifier as shown in Fig. 7.

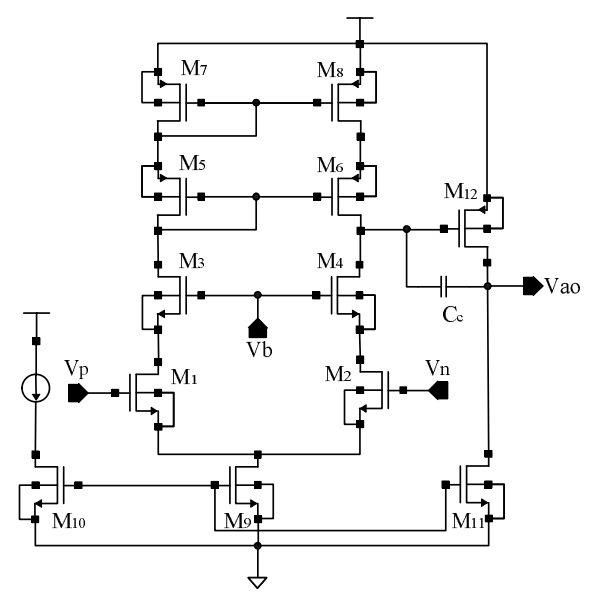

Fig. 7 Telescopic cascode operational amplifier circuit

If $(W / L)_{2}=(W / L)_{4}$ and $(W / L)_{8}=(W / L)_{6}$, the voltage gain of the first stage op amp and the second stage op amp is

$$
\begin{aligned}
A_{1} & =g_{m 1} \cdot\left(g_{m 2} r_{o 2}{ }^{2} \mathrm{P} g_{m 8} r_{o 8}{ }^{2}\right) \\
A_{2} & =g_{m 12} r_{o 12}
\end{aligned}
$$

Where $g_{m}$ and $r_{o}$ are transconductance and resistance of transistor. Therefore, the open-loop gain of the operational amplifier is

$$
A=A_{1} \cdot A_{2}=g_{m 1} g_{m 12} \cdot\left(g_{m 2} r_{o 2}{ }^{2} \mathrm{P} g_{m 8} r_{o 8}{ }^{2}\right) r_{o 12}
$$

\section{Simulation result}

Based on TSMC $0.18 \mu \mathrm{m}$ CMOS process, the readout circuit is simulated by Spectre of Cadence software. The circuit uses a single $3.3 \mathrm{~V}$ power supply. The simulation results of telescopic cascode operational amplifier that the open-loop gain is $82 \mathrm{~dB}$, the unity gain bandwidth is $35 \mathrm{MHz}$, the phase margin is $63.5^{\circ}$, the common mode rejection ratio is $73.2 \mathrm{~dB}$, the input referred noise $(1 \mathrm{KHz})$ is $125 \mathrm{nV} / \sqrt{\mathrm{Hz}}$. In the readout circuit as shown in Fig. 3, $C_{m}=600 \mathrm{fF}, C_{r e f}=300 \mathrm{fF}, C_{s}=330 \mathrm{fF}$, the voltage simulation results are shown as Fig. 8. 


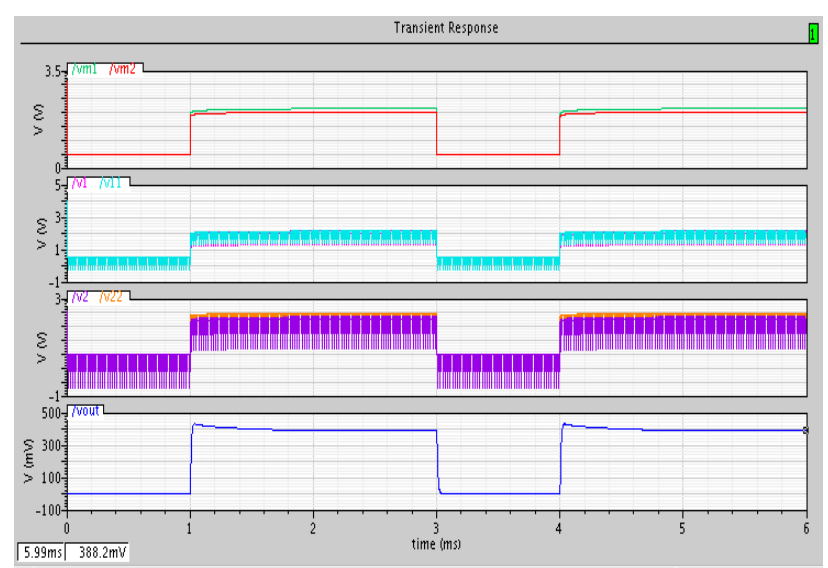

Fig. 8 The readout circuit simulation results

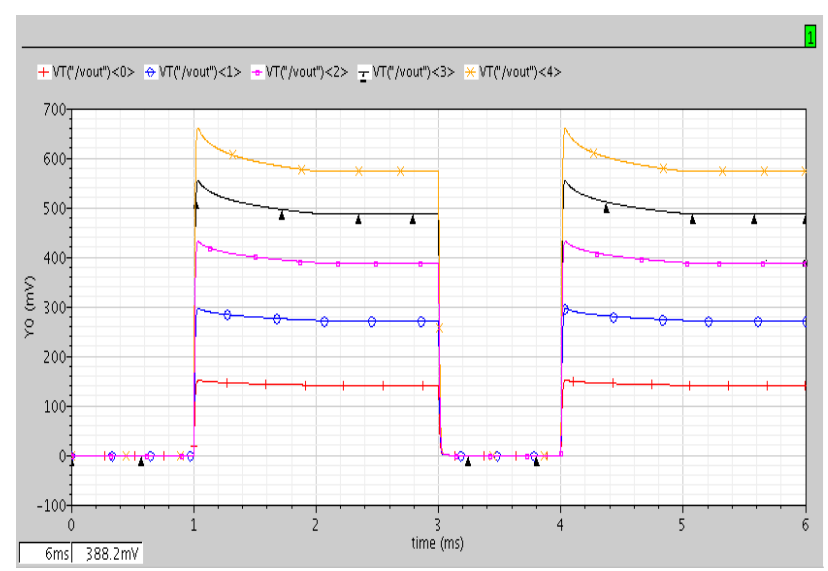

Fig. 9 The output voltage simulation results

When the capacitance changes of $C_{s}$ from $310 \mathrm{fF}$ to $350 \mathrm{fF}$, the results show that the output voltage changes from $142 \mathrm{mV}$ to $572 \mathrm{mV}$ in Fig. 9 . The sensitivity of the converter is $10.8 \mathrm{mV} / \mathrm{fF}$. The readout circuit can remove the effect of DC offset.

\section{Conclusions}

The readout circuit for capacitive $\mathrm{pH}$ sensor is designed and implemented using the technique of chopper stabilization and instrumentation amplifier in the paper. The high sensitivity, low noise and low offset performance of the readout circuit is confirmed with Cadence software and TSMC $0.18 \mu \mathrm{m}$ 3.3V CMOS process technology simulaions.

\section{Acknowledgments}

This work was supported by Zhejiang Province Natural Science Foundation of China (LQ16F010005, LY14F040002), and Ningbo Natural Science Foundation (2013A610010, 2013A610008).

\section{References}

[1] P.K.Rathore and B.S.Panwar: IEEE Multi-Conference on Systems and Control (2013), p.443448.

[2] M.Narducci, L.Yuchia, W.Fang and J.Tsai: Journal of Micromechanics \& Microengineering Vol. 23 (2013), p.55007-55011.

[3] P. Bergveld: Sensors \& Actuators B Vol. 88 (2003), p.1-20.

[4] S.Martinoia, G.Massobrio and L.Lorenzelli: Sensors \& Actuators B Vol. 105 (2005), p.14-27.

[5] P.Georgiou and C.Toumazou: Sensors \& Actuators B Vol.143 (2009), p.211-217.

[6] Md.S.Arefin, M.B.Coskun, T.Alan, J.M.Redoute, A.Neild and M.R.Yuce: Applied Physics Letters 104, 223503 (2014).

[7] J.Shiah, H.Rashtian and S.Mirabbasi: IEEE International Symposium on Circuits \& Systems (2010), p.3280-3283.

[8] F.Butti, P.Bruschi, M.Piotto: Conference on Phd Research in Microelectronics \& Electronics (2011), p.133-136.

[9] M.Piotto, F.Butti, A.D.Pancrazio and P.Bruschi: Procedia Engineering Vol. 87 (2014), p.736739.

[10] P.D.Dimitropoulos, D.P.Karampatzakis, G.D.Panagopoulos and G.I.Stamoulis: Sensors Journal IEEE Vol. 6 (2006), p.755-769.

[11] R.Schinzinger and P.A.A.Laura: Ocean Engineering (2004), p.17-18.

[12] I.Evans, A.Somerville and T.York: 1st world congress on industrial process tomography (1999), p.395-401. 